\title{
Prevalence and determinants of alcohol use among adults living with HIV/AIDS in Ethiopia: a systematic review protocol
}

\author{
Birhanie Mekuriaw $^{1^{*}}$ (D) Zelalem Belayneh ${ }^{1}$, Alemayehu Molla ${ }^{1}$ and Tsegaye Mehare ${ }^{2}$
}

\begin{abstract}
Background: Alcohol use is a challenging problem which attributes to more than $5 \%$ of the overall global burden of disease. It is more common among persons with HIV infection than the general population. Although there are separate studies regarding people with HIV/AIDS in Ethiopia, their results are highly variable and discrepant. The objectives of this study will be to evaluate the prevalence of alcohol use and to identify its associated factors among people with HIV/AIDS in Ethiopia.

Methods: A systematic search of electronic databases (from inception onwards) of PubMed/MEDLINE, Embase, PsycINFO, and Cochrane Library will be conducted. Moreover, grey literatures will be searched from different sources (such as Google Scholar, OpenGrey, and World Health Organization websites). Reference lists of the selected articles will also be searched manually. Observational studies (cross-sectional, case-control, cohort) reporting the prevalence of alcohol use and/or its associated factors among adults with HIV/AIDS in Ethiopia will be included. The primary outcomes will be the prevalence of alcohol use among HIV/AIDS population. Secondary outcomes will be the determinants of alcohol use described in the included studies. Two reviewers will independently screen all citations and full-text articles and extract data. The studies' methodological quality (or bias) will be appraised using an appropriate tool. If feasible, we will conduct a random effects meta-analysis of observational data. Heterogeneity of primary studies will be assessed using the $P^{2}$ test. Prevalence estimates will be stratified according to gender, age, and geographical location. Small-study effects (publication bias) also will be examined.

Discussion: Our systematic review and meta-analysis will prevail the pooled prevalence of alcohol use and its determinants among people with HIV/AIDS in Ethiopia. The finding of this study will be helpful to design appropriate preventive and interventional strategies for alcohol use among people with HIV/AIDS. This can have direct or indirect policy responses and clinical implications.
\end{abstract}

\section{Systematic review registration: PROSPERO CRD42019132524}

Keywords: Prevalence, Harmful drinking, Alcohol use, Substance use, Addiction, Drinking habit, HIV/AIDS, Ethiopia

\footnotetext{
*Correspondence: biradilla@gmail.com

'Department of Psychiatry, College of Health and Medical Science, Dilla University, Dilla, Ethiopia

Full list of author information is available at the end of the article
}

(c) The Author(s). 2020 Open Access This article is licensed under a Creative Commons Attribution 4.0 International License, which permits use, sharing, adaptation, distribution and reproduction in any medium or format, as long as you give appropriate credit to the original author(s) and the source, provide a link to the Creative Commons licence, and indicate if changes were made. The images or other third party material in this article are included in the article's Creative Commons licence, unless indicated otherwise in a credit line to the material. If material is not included in the article's Creative Commons licence and your intended use is not permitted by statutory regulation or exceeds the permitted use, you will need to obtain permission directly from the copyright holder. To view a copy of this licence, visit http://creativecommons.org/licenses/by/4.0/ The Creative Commons Public Domain Dedication waiver (http://creativecommons.org/publicdomain/zero/1.0/) applies to the data made available in this article, unless otherwise stated in a credit line to the data. 


\section{Background}

Alcohol use is an important public health problem with higher morbidity and mortality rate. According to the World Health Organization (WHO) report, more than 3 million people died each year due to harmful alcohol consumption [1, 2]. Alcohol is considered the third leading cause of death that contributes to $5 \%$ of the overall global burden of disease [3]. The morbidity and mortality rates attributed to alcohol are expected to be greater than the combined numbers of deaths due to human immunodeficiency virus (HIV), acquired immunodeficiency syndrome (AIDS), violence, and tuberculosis globally $[4,5]$.

A bunch of reasons has been identified as contributing factors to the highly prevalent alcohol use problem among people with human immunodeficiency virus/acquired immunodeficiency syndrome (HIV/AIDS) [6, 7]. Some studies showed that medication nonadherence, psychological distress, poor quality of life, family history of alcohol or other substance use, poor coping skills to accept HIV-positive serostatus, and poor social support can contribute to harmful alcohol consumption among people living with HIV/AIDS [8-11].

Despite the high prevalence of alcohol use, there is still a challenge that alcohol or other substance users mostly underreport or totally deny their consumption due to social taboos and its conflict with medical advices. This needs to design strategies with a multi-sectoral effort to elicit the true picture of harmful alcohol consumption which negatively affects the treatment outcomes of HIV/AIDS care [12, 13].

Alcohol use can have negative effects on the treatment outcome, disease progression, and overall quality of life of people with HIV/AIDS [14]. For example, alcohol increases treatment delay, reduces adherence and treatment compliance, fastens disease progression, reduces viral load suppression, and negatively affects the person's functionality. Individuals with alcohol use problems also often engage in polysubstance use and other risky behaviors like multiple partners and unsafe sex which may in turn result in increased risks of further HIV transmission $[15,16]$.

In Ethiopia, there are different traditional homemade alcoholic beverages (Tella, Tej, Arki, Wine) with different and unestimated alcoholic contents. People commonly use such alcoholic beverages in different parties, ceremonies, and family preparations. In some rural communities of Ethiopia, such homemade traditional alcoholic beverages are consumed on a daily basis, and alcohol is considered as a socially acceptable drink including during their meals $[8,17$, 18]. As a result, individuals with HIV/AIDS may use these locally available alcoholic beverages as pain killer, to induce sleep, as a means for social engagement, and to escape from their stressful environment during a crisis.

Although some studies have existed regarding alcohol use among adults with HIV/AID in Ethiopia, there is a great discrepancy and inconsistency of the reported results. For instance, a study conducted in Zewuditu Memorial Hospital showed the lowest prevalence (6.5\%) of alcohol use, and the highest prevalence (32.6\%) was observed from a study conducted in Jimma $[8,19]$. This demonstrates a need for the full picture of the problem to develop effective intervention and for policy responses that can support decreasing alcohol consumption among the HIV/AIDS population. Therefore, the objectives of this systematic review will be to evaluate the prevalence of alcohol use and to identify its associated factors among adults with HIV/AIDS in Ethiopia.

\section{Methods}

This study protocol is being reported in accordance with the reporting guidance provided in the Preferred Reporting Items for Systematic Reviews and Meta-analysis Protocols (PRISMA-P) statement [20, 21] (see Additional file 1). The review protocol has been registered in the International Prospective Register of Systematic Reviews (PROSPERO) [CRD42019132524].

\section{Information sources and search strategy}

A systematic search of PubMed/MEDLINE, Embase, PsycINFO, and Cochrane Library will be conducted from inception onwards. The search strategy will be developed in consultation with a health sciences librarian, which will be reviewed using the Peer Review of Electronic Search Strategies (PRESS) Checklist [22]. The search strategy will be developed in PubMed/MEDLINE (see Additional file 2) and adapted to the other bibliographic databases. Search terms will include subject headings (e.g., MeSH in PubMed/MEDLINE) for each database and free-text words for the key concepts of "prevalence," "epidemiology," "alcohol use," "HIV," "AIDS," and "Ethiopia." Grey or difficult to locate literature will be searched, including Google Scholar, Open Grey, and the World Health Organization (WHO) websites. We will also search reference lists of included studies and related reviews.

\section{Inclusion criteria}

Studies will be selected according to the following criteria: study design, participants, settings, and outcomes of interest.

\section{Study design}

Observational studies (cross-sectional, case-control, and cohort studies) reporting the prevalence and/or associated factors of alcohol use among adults with HIV/AIDS will be included.

\section{Population}

Primary studies conducted among adults (age $\geq 18$ ) living with HIV/AIDS will be considered. 


\section{Settings}

Studies conducted in a wide range of HIV people from the community and/or outpatient settings in any Ethiopian region will be considered.

\section{Outcomes of interest}

The primary outcome will be the prevalence of alcohol use indicating the number of HIV/AIDS people that consume alcohol divided by the HIV/AIDS adult population number at a given point in time. This is often presented as a prevalence (proportion). We will use authorreported definitions according to accepted standardized measurement tools or questionnaires such as Alcohol Use Disorder Identification Test (AUDIT); Cut down, Annoying, Guilt feeling, and Eye Opener (CAGE); or other important measurement tools used to determine the level of alcohol consumption. The secondary outcomes will be the determinants or associated factors of alcohol use reported in the included studies (such as gender, marital status, smoking behavior, mental distress, social support level, and khat chewing habit). No limitations will be imposed on publication status (unpublished studies will be eligible for inclusion) and study conduct period. Only studies published in English (or with an English translation available) will be considered.

\section{Screening and selection of studies}

All articles identified from the literature search will be screened by two reviewers (ZB and BM), independently after duplicate removal by using EndNote. Titles and abstracts of articles returned from the initial searches will be screened based on the eligibility criteria outlined above (the "Inclusion criteria" section). Full texts will be examined in detail and screened for eligibility. References of all considered articles will be hand-searched to identify any relevant report missed in the search strategy. Any disagreements will be resolved by a discussion, if necessary. A PRISMA flow chart showing details of studies included and excluded at each stage of the study selection process will be provided.

\section{Data abstraction}

A data extraction form will be designed in Microsoft Excel and used by two reviewers (BM and $\mathrm{ZB}$ ) to extract equivalent information from each study report. A single row will be used for each study. Information of interest will include the following.

\section{Study characteristics}

The following are the study characteristics: name of the first author, study design, year of publication, journal, year (or period) of study conduct, sample size, setting, geographical location where the study is conducted, and other fields to capture data relevant to the assessment of study methodological quality.

\section{Participant characteristics}

The following are the participant characteristics: population sampled, age (e.g., mean with standard deviation, range), and gender (e.g., percentage of female and male participants).

\section{Outcome results}

Definitions and measures used to make diagnosis (of alcohol use and the determinants of use), prevalence estimates (e.g., number of subjects with the outcome, proportion, and 95\% confidence interval), and any prevalence estimates stratified by age, gender, or study location. For the second outcome (determinants of alcohol use), important data will be extracted using two by two tables for each predictor. Any disagreement between the two authors during data extraction will be solved through a discussion.

\section{Quality assessment}

Two authors will independently assess the overall qualities of primary articles using the Newcastle-Ottawa Scale quality assessment tool [23, 24]. The tool has three different sections. The first section assesses the methodological quality of each study, the second part examines the comparability of primary studies, and the last part assesses articles with respect to their statistical analysis and interpretation. Any disagreements between the two assessors are expected to be negotiated through a discussion and by taking the average score of the two different assessment results. Articles fulfilling equal to or greater than $50 \%$ of the quality assessment criteria score will be included in this review.

\section{Data synthesis}

The data from each study will be used to build evidence tables of an overall description of the included studies. Crude prevalence estimates (number of cases/sample size) will be presented along with $95 \%$ confidence intervals. If feasible and appropriate, prevalence data points from primary studies will be used to perform random effects meta-analyses. Since heterogeneity is expected, we will estimate the pooled prevalence and its 95\% confidence interval using the random effects model. The random effects model [25] assumes the study prevalence estimates follow a normal distribution, considering both within-study and between-study variations. We will also conduct a quality-effects model to examine how the quality of each study changed the pooled estimate compared with the results from the random-effects metaanalysis [26]. Forest plots will be used to visualize pooled estimates and the extent of heterogeneity among studies.

We will quantify statistical heterogeneity by estimating the variance between studies using the $I^{2}$ statistic [27]. 
The $I^{2}$ statistic is the proportion of variation in prevalence estimates that is due to the genuine variation in prevalence rather than sampling (random) error. The $I^{2}$ statistic ranges between 0 and 100\% (with values of $0-25 \%$ and 75-100\% taken to indicate low and considerable heterogeneity, respectively). We will also report the Cochran $Q$ test with a $p$ value of $<0.05$ considered statistically significant. All statistical analyses will be performed using Stata14 (StataCorp LP, College Station, TX, USA)

If sufficient studies are identified and data points are available, potential sources of heterogeneity will be investigated further by subgroup analyses according to baseline characteristics and methodological covariates. We plan to conduct analyses by gender (male vs. female), geographical location of studies (e.g., using regions where the studies are conducted), study quality (e.g., low vs. moderate/high quality), and publication year.

Small study effects (publication bias across studies) will be assessed by inspection of the funnel plots for asymmetry and Egger's test [28-30], with the results considered to indicate potential small study effects when $p$ values are $<0.10$.

\section{Discussion}

This systematic review and meta-analysis will determine the prevalence of alcohol use among HIV/AIDS patients in Ethiopia. In addition, this study will identify factors associated with alcohol use among people with HIV/AIDS. Even though there are empirical evidences about the magnitude of alcohol use and preventive measures, it remains still a challenging problem in the management of people with HIV/AIDS. The findings of this review could be an important input in health policy and planning debates, such as the development of evidence-based prevention and intervention programs for people with HIV/AIDS. This review will identify and fill the knowledge gaps in the field with new research. On this regard, implications for future observational studies will be discussed.

The proposed systematic review and meta-analysis will be reported in accordance with the reporting guidance provided in the Preferred Reporting Items for Systematic Reviews and Meta-analyses (PRISMA) statement [20, 21] and the Meta-analysis Of Observational Studies in Epidemiology (MOOSE) reporting guideline [31]. Any amendments made to this protocol during the study conduct will be reported in the final manuscript and reported in PROSPERO. Results will be disseminated through publication in a peer-reviewed journal.

There are several limitations to our planned systematic review. We anticipate identifying observational studies conducted in Ethiopia with different study designs, populations, and contexts and with a variable quality of reporting of methods and results. In addition, publication and reporting biases can distort the planned review and meta-analysis.

\section{Supplementary information}

Supplementary information accompanies this paper at https://doi.org/10. 1186/s13643-020-01402-w.

Additional file 1. PRISMA-P Checklist.

Additional file 2. Search strategy for PubMed.

\section{Abbreviations}

HIV/AIDS: Human immunodeficiency virus /acquired immunodeficiency syndrome; MOOSE: Meta-analysis Of Observational Studies in Epidemiology; PRESS: Peer Review of Electronic Search Strategies; PRISMA-P: Preferred Reporting Items for Systematic Reviews and Meta-analysis; WHO: World Health Organization

Acknowledgements

Not applicable

Authors' contributions

BM conceived the idea and designed the study. BM and ZB drafted the first protocol. AM and TM reviewed the protocol. All authors read and approved the final draft of the protocol.

\section{Funding}

Not applicable

Availability of data and materials Not applicable.

Ethics approval and consent to participate

Not applicable.

Consent for publication

Not applicable.

Competing interests

All authors declare that they have no competing interests.

\section{Author details}

${ }^{1}$ Department of Psychiatry, College of Health and Medical Science, Dilla University, Dilla, Ethiopia. ${ }^{2}$ Department of Biomedical Science, College of Health and Medical Science, Dilla University, Dilla, Ethiopia.

Received: 10 June 2019 Accepted: 29 May 2020

Published online: 08 June 2020

\section{References}

1. Chander G, Josephs J, Fleishman J, Korthuis PT, Gaist P, Hellinger J, Gebo K, Network HR. Alcohol use among HIV-infected persons in care: results of a multi-site survey. HIV Medicine. 2008;9(4):196-202.

2. Fisher JC, Bang H, Kapiga SH. The association between HIV infection and alcohol use: a systematic review and meta-analysis of African studies. Sexually Transmitted Diseases. 2007;34(11):856-63.

3. Mathers C, Ayuso-Mateos JL: Global burden of alcohol use disorders in the year 2000: summary of methods and data sources. In.: Citeseer; 2003.

4. Unit WHOMoSA: Global status report on alcohol and health, 2014: World Health Organization; 2014

5. Poznyak V, Fleischmann A, Rekve D, Rylett M, Rehm J, Gmel G. The World Health Organization's global monitoring system on alcohol and health. Alcohol Research: Current Reviews. 2014;35(2):244.

6. Samet JH, Horton NJ, Meli S, Freedberg KA, Palepu A. Alcohol consumption and antiretroviral adherence among HIV-infected persons with alcohol problems. Alcoholism: Clinical and Experimental Research. 2004;28(4):572-7.

7. Kalichman SC, Simbayi LC, Kaufman M, Cain D, Jooste S. Alcohol use and sexual risks for HIV/AIDS in sub-Saharan Africa: systematic review of empirical findings. Prevention Science. 2007;8(2):141.

8. Soboka M, Tesfaye M, Feyissa GT, Hanlon C. Alcohol use disorders and associated factors among people living with HIV who are attending services in south west Ethiopia. BMC Research Notes. 2014;7(1):828.

9. Bultum JA, Yigzaw N, Demeke W, Alemayehu M. Alcohol use disorder and associated factors among human immunodeficiency virus infected patients 
attending antiretroviral therapy clinic at Bishoftu General Hospital, Oromiya region, Ethiopia. PloS One. 2018;13(3)::0189312. https://doi.org/10.1371/ journal.pone.0189312.

10. Catz SL, Gore-Felton C, McClure JB. Psychological distress among minority and low-income women living with HIV. Behav Med. 2002;28(2):53-60.

11. Kaplan B, Sadock V: Synopsis of psychiatry: updated with DSM-5. In.: Wolter Kluwer: Amazon; 2015

12. Sadok B, Sadok V, Pedro R: Kaplan and Sadock's synopsis of psychiatry. Updated with DSM-5 2015.

13. Shim R, Rust G: Primary care, behavioral health, and public health: partners in reducing mental health stigma. In:: American Public Health Association; 2013.

14. Belayneh Z, Alemu W, Mekuriaw B, Abebe Z. Bipolar spectrum disorders and associated factors among adults attending an antiretroviral therapy clinic in gedeo zone health centers, southern ethiopia. Neuropsychiatr Dis Treat. 2019;15:503.

15. Morojele NK, Kachieng'a MA, Mokoko E, Nkoko MA, Parry CD, Nkowane AM, Moshia KM, Saxena S. Alcohol use and sexual behaviour among risky drinkers and bar and shebeen patrons in Gauteng province, South Africa. Soc Sci Med. 2006;62(1):217-27.

16. Shuper PA, Joharchi N, Irving H, Rehm J. Alcohol as a correlate of unprotected sexual behavior among people living with HIV/AIDS: review and meta-analysis. AIDS and Behavior. 2009;13(6):1021-36.

17. Mekuriaw B, Belayneh Z, Shemelise T, Hussen R. Alcohol use and associated factors among women attending antenatal care in Southern Ethiopia: a facility based cross sectional study. BMC Research Notes. 2019;12(1):690.

18. Mekuriaw B, Belayneh Z, Yitayih Y. Magnitude of Khat use and associated factors among women attending antenatal care in Gedeo zone health centers, southern Ethiopia: a facility based cross sectional study. BMC Public Health. 2020;20(1):110.

19. Gebremariam EH, Reta MM, Nasir Z, Amdie FZ. Prevalence and associated factors of suicidal ideation and attempt among people living with HIV/AIDS at Zewditu Memorial Hospital, Addis Ababa, Ethiopia: a cross-sectional study. Psychiatry J. 2017;2017.

20. Moher D, Shamseer L, Clarke M, Ghersi D, Liberati A, Petticrew M, Shekelle P, Stewart LA. Preferred Reporting Items for Systematic Review and MetaAnalysis Protocols (PRISMA-P) 2015 statement. Syst Rev. 2015;4(1):1.

21. Shamseer L, Moher D, Clarke M, Ghersi D, Liberati A, Petticrew M, Shekelle P, Stewart LA. Preferred reporting items for systematic review and meta-analysis protocols (PRISMA-P) 2015: elaboration and explanation. BMJ. 2015;349.

22. McGowan J, Sampson M, Salzwedel DM, Cogo E, Foerster V, Lefebvre C PRESS peer review of electronic search strategies: 2015 guideline statement. Journal of Clinical Epidemiology. 2016;75:40-6.

23. Newcastle-Ottawa Scale adapted for cross-sectional studies, https:// wellcomeopenresearch.s3.amazonaws.com/supplementary/13880/ea30a2fba15a-44a9-b35e-5f0914db80b3.docx.

24. Peterson J, Welch V, Losos M, Tugwell P. The Newcastle-Ottawa scale (NOS) for assessing the quality of nonrandomised studies in meta-analyses. Ottawa: Ottawa Hospital Research Institute; 2011

25. Berkey CS, Hoaglin DC, Mosteller F, Colditz GA. A random-effects regression model for meta-analysis. Statistics in Medicine. 1995;14(4):395-411.

26. Doi SA, Thalib L. A quality-effects model for meta-analysis. Epidemiology. 2008:94-100.

27. Higgins JP, Thompson SG. Quantifying heterogeneity in a meta-analysis. Stat Med. 2002;21(11):1539-58.

28. Liu JL. The role of the funnel plot in detecting publication and related biases in meta-analysis. Evi Based Dent. 2011;12(4):121-2.

29. Egger M, Smith GD, Schneider M, Minder C. Bias in meta-analysis detected by a simple, graphical test. BMJ. 1997;315(7109):629-34.

30. Sterne JA, Egger M. Funnel plots for detecting bias in meta-analysis: guidelines on choice of axis. J Clin Epidemiol. 2001;54(10):1046-55.

31. Stroup DF, Berlin JA, Morton SC, Olkin I, Williamson GD, Rennie D, Moher D, Becker BJ, Sipe TA, Thacker SB. Meta-analysis of observational studies in epidemiology: a proposal for reporting. JAMA. 2000;283(15):2008-12.

\section{Publisher's Note}

Springer Nature remains neutral with regard to jurisdictional claims in published maps and institutional affiliations.

\section{Ready to submit your research? Choose BMC and benefit from:}

- fast, convenient online submission

- thorough peer review by experienced researchers in your field

- rapid publication on acceptance

- support for research data, including large and complex data types

- gold Open Access which fosters wider collaboration and increased citations

- maximum visibility for your research: over $100 \mathrm{M}$ website views per year

At $\mathrm{BMC}$, research is always in progress.

Learn more biomedcentral.com/submissions 